\title{
Adrenergic and cholinergic innervation of the atrioventricular valves in chinchilla (Chinchilla laniger)
}

\author{
J. Kuchinka1, M. Chrzanowska1, T. Kuder² \\ ${ }^{1}$ Department of Comparative Anatomy, Institute of Biology, Jan Kochanowski University, Kielce, Poland \\ ${ }^{2}$ Department of Normal and Functional Anatomy, Institute of Medical Sciences, Jan Kochanowski University, Kielce, Poland
}

[Received 10 January 2017; Accepted: 23 March 2017]

The arrangement of autonomic fibres was studied in the cardiac atrioventricular valves of small chinchillas. The dissected valves were stained entirely using the modified histochemical acetylcholine esterase technique (AChE) and the SPG-De la Torre method. Double immunocytochemical staining was also used for the expression of vesicular acetylcholine transporter (VAChT) and dopamine beta hydroxylase $(D B H)$. The study showed the presence of both cholinergic and adrenergic fibres, forming a kind of network on all cusps of both valves. The adrenergic network is always more strongly represented than the cholinergic network. The cholinergic nerve network of the "parietal" part formed mainly the parallel arrangement. As we move towards the free parts of the cusps, the arrangement becomes netted and radiant. The adrenergic fibres formed only the netted arrangement, which was the most dense in the peripheral (parietal) part of the cusps. Some of the fibres in the vicinity of tendinous cords extended as far as the papillary muscles. Double immunocytochemical tests confirmed the presence and distribution of DBH-and VAChT-positive fibres. Some fibres (especially within the tendinous cords) show VAChT and DBH colocalisation. (Folia Morphol 2017; 76, 4: 590-595)

Key words: atrioventricular valves, autonomic innervations, chinchilla

\section{INTRODUCTION}

Because of their very important role in the proper functioning of the heart, the atrioventricular valves are the subject of interest of many authors.

It is known that, in addition to fibroblast cells, the endocardium may also contain myocytes. These are supplied by the sympathetic and parasympathetic nerve fibres [2, 14, 16, 23, 25]. Additionally, there are reports of the presence of neuropeptides: neuropeptide Y (NPY), vasoactive intestinal peptide (VIP), substance $P(S P)$ and nitric oxide synthase (NOS) $[1,10]$.

As shown in dogs, the mitral valve displays spontaneous contractility after electrical stimulation. It was observed that after the addition of noradrenaline (NA), its activity increased, while after the adminis- tration of acetylcholine esterase (AChE) it decreased $[6,24]$.

Due to the fact that the literature does not contain data on the innervation of the heart valves in chinchillas, which are farm animals, this study was undertaken to understand the innervation of atrioventricular valves in small rodents. Apart from their cognitive value, the results may also have a practical aspect.

\section{MATERIALS AND METHODS}

The valves (mitral and tricuspid) were isolated from the hearts collected from 5 carcasses of adult animals of both sexes acquired during industrial slaughter (Chinchillas Fur Farm in Kielce). This study was approved in accordance with the law of Act of 
Table 1. Antibody used in the study

\begin{tabular}{|c|c|c|c|c|c|}
\hline Primary antibody & & & & & \\
\hline Antigen & Host & Type & Dilution & Cat. No & Supplier \\
\hline DBH & Mouse & Monoclonal & $1: 500$ & MAB308 & Milipore \\
\hline VAChT & Rabbit & Polyclonal & $1: 5000$ & V5387 & Sigma \\
\hline \multicolumn{6}{|l|}{ Secondary antibody } \\
\hline Host & & Fluorochrome & Dilution & Code & Supplier \\
\hline Goat-anti-mouse IG (H+L) & & AlexaFlour 485 & $1: 500$ & A11001 & Invitrogen \\
\hline Goat-anti-rabbit IG $(\mathrm{H}+\mathrm{L})$ & & AlexaFlour 568 & $1: 500$ & A11011 & Invitrogen \\
\hline
\end{tabular}

DBH — dopamine beta hydroxylase; VAChT — vesicular acetylocholine transporter

15 January 2015, "The protection of animals used for scientific or educational purpose" (studies on tissues obtained post-mortem do not require an approval of the Ethics Committee).

The abdominal cavity and chest was opened as soon as possible. Heart perfusion was performed via abdominal aorta by $4 \%$ ice-cold buffered paraformaldehyde. The valve cups along with annuli fibrosi, tendinous cords and papillary muscle were isolated. The dissected valves were stained entirely using the modified histochemical AChE technique created by Gienc [7] and the sucrose-potassium phosphate-glyoxylic acid (SPG) histofluorescence method for the visualisation of biogenic monoamines, developed by De la Torre [5]. The adrenergic items were identified using glyoxylic acid (A 16058 - Alfa Aesar Germany) method (De la Torre, 1980). The obtained histological and histochemical slides were examined using a stereomicroscope (Nikon SMZ-800, Japan) and advanced automated research microscope, Nikon Eclipse 90i (Japan) with integrated epi-fluorescence attachment (Filters: DAPI EX 359 nm, EM 461 nm; FITC EX 490 nm, EM 525 nm; CY3 EX 552 nm, EM 570 nm). Digital photographs were taken with a Nikon Digital Sight SD-LI-Japan camera system and calculations were carried out using Imaging Software NIS Elements - Advanced Solutions for Imaging World (version 4.11). The density of nerve fibres was taken from $\mathrm{N}+10$ randomly chosen regions $\left(0.5 \mathrm{~mm}^{2}\right)$ of the mitral and tricuspid valves.

Double immunocytochemical staining was also used for the expression of vesicular acetylcholine transporter (VAChT) and dopamine beta hydroxylase (DBH). The whole valves were washed $3 \times 10 \mathrm{~min}$ in phosphate buffer (PB), incubated for $45 \mathrm{~min}$ in 10\% normal goat serum (NGS, Cappel, Poland) in PB containing $0.25 \%$ Triton X-100 (Sigma, USA) and then incubated over- night at room temperature with antibodies (Table 1) diluted in PB containing $0.25 \%$ Triton X-100. After incubation with primary antibody, the sections were washed $3 \times 10$ min in PB and further incubated with secondary antibodies for $1 \mathrm{~h}$ in room temperature. After incubation, the sections were washed $3 \times 10$ min in PB, coverslipped with buffered glycerol and examined under a research microscope, Nikon Eclipse 90i (Japan). The control of specificity of antisera was performed by preabsorbtion of a diluted antibody with $20 \mu \mathrm{g} / \mathrm{mL}$ of an appropriate antigen, which completely abolished the specific immunoreaction. In addition, the primary antibody was replaced by non-immune serum or by $\mathrm{PB}$ in order to verify the specificity of particular immunoreactions.

\section{RESULTS}

The study showed the presence of both cholinergic and adrenergic fibres, forming a kind of network on all cusps of both valves. The adrenergic network is always more strongly represented than the cholinergic network. This was confirmed by double immunocytochemical tests.

AChE-positive fibres. An intensely stained cholinergic neural network was observed in the immediate vicinity of the annulus fibrosus of the two valves; it consisted of 4-5 nerve fibres forming an approximately parallel arrangement with respect to the annuli fibrosi. There were transverse branches among them. Numerous branches branched off from this "parietal plexus" towards the free edges of the valve cusps, forming a fairly dense nervous plexus (45-65 nerve fibres $/ 0.5 \mathrm{~mm}^{2}$ ) in which fibres with a radial arrangement prevailed. The network had polygonal holes in the central part of the cusps, while the "bushy" arrangement predominated in the peripheral portion. The degree of density and the arrangement 
of fibres were very similar in all cusps of the two valves (Figs. 1, 2). As we moved towards the edge of the valve cusps, the density of the plexus decreased significantly (15-23 nerve fibres/0.5 $\mathrm{mm}^{2}$ ). Only a small part of the fibres in the vicinity of tendinous cords extended as far as the papillary muscles.

SPG-positive fibres. The entire surface of the valve cusps displayed a dense adrenergic network (Figs. 3, 4). The largest concentration of fibres was in the immediate vicinity of the annulus fibrosus with no parallel peripheral fibres. It was 78-119 nerve fibres per $0.5 \mathrm{~mm}^{2}$. As we moved towards the edge of the cusps, the neural network became increasingly thin $\left(49-65 / 0.5 \mathrm{~mm}^{2}\right)$. The holes in the network were polygonal and varied in size, and the fibres were undulating. Careful analysis allowed us to distinguish both the superficial and the deep network systems. The superficial arrangement passes to the tendinous cords and papillary muscles. On the surface of the tendinous cords, the fibres were mainly parallel (Fig. 5).

Immunocytochemistry. Immunocytochemical tests for VAChT- and DBH-positive fibres confirmed the arrangement of adrenergic and cholinergic fibres detected by classic histochemical techniques. The arrangement of VAChT-immunoreactive fibres in the "parietal" region was mainly parallel with respect to the fibrous ring. In the central part of the cusps, it created a highly-branched, polygonal bushy network. The "marginal" part of the cusps mainly displayed a radial arrangement, which passed on the tendinous cords, forming there a mostly parallel arrangement.

The DBH-immunoreactive fibres were significantly more strongly represented than VAChT-positive fibres (2 up to 3 times more). The arrangement of these fibres on the whole surface of the valve cusps was netted. In the marginal zone its intensity decreased and some fibres in this way reached the tendinous cords. Here and there in the valve cusps, there was VAChT and DBH colocalisation of fibres (Fig. 6). This was especially pronounced in the "parachordal" part of the cusps and within the tendinous cords.

\section{DISCUSSION}

The innervation of cardiac atrioventricular valves was studied in several animal species, especially laboratory animals - mice, rats, guinea pigs, rabbits, opossums [1, 2, 8, 23, 25] and in domestic animals - cats, dogs and pigs $[3,4,17,23]$. Human hearts were also studied in this respect $[4,15,16,22]$.
As these studies clearly indicate, the density of neural networks and their distribution is diverse and largely depend on species of study animals. Thus, for example, Williams et al. [25] showed that nerve fibres in the valves of mice, guinea pigs and opossum create denser plexuses than in rats. Within the same species, a relationship was observed with the age of an individual. The studies conducted by Smith [23] showed the absence of nerve fibres at the edges of the cusps of the atrioventricular valves in elderly individual guinea pigs, while Folan-Curran et al. [8] observed the presence of nerve fibres in young individuals of this species.

Borin et al. [2] showed that the mitral valve in mice, rats, guinea pigs, and opossum is more strongly innervated than the tricuspid valve. On the other hand, Kumar and Tay [13] demonstrated that the tricuspid valve in rats and rabbits was more strongly innervated than the mitral valve. According to Marron et al. [16], in human the anterior cusp of the mitral valve has a network of nerve fibres that is twice as dense as that of the posterior cusp.

The results of this study indicated that the arrangement of adrenergic and cholinergic fibres, and the degree of their density, was similar in all the cusps, both in the right and left heart. It seems, therefore, that these differences in the innervation of individual valves can be explained by large interspecies variations, which are a specific feature of the autonomic nervous system. This interpretation is also supported by the fact that, despite some interspecies differences, we can demonstrate some common features in the innervation of the mitral and tricuspid valve in many animal species. These include features such as the largest concentration of the network of nerve fibres in the immediate vicinity of the fibrous ring and the closer the free edges of the cusps are, the thinner this network becomes $[2,25]$. The study confirmed these reports with respect to chinchillas, in which the cholinergic network was more strongly represented in the immediate vicinity of the fibrous ring on the cusps of both valves. It consisted of 4-5 nerve fibres, forming a parallel arrangement in relation to the annuli. Both in chinchillas and other species, many nerve branches branch off from the "para-rings" fibres, pointing toward the free edges of the valve cusps. In the central part of the cusps, they form polygonal holes, while the peripheral portion they have a mainly radial arrangement. Interspecies differences were evident in reaching the free edges by nerve fibres: 


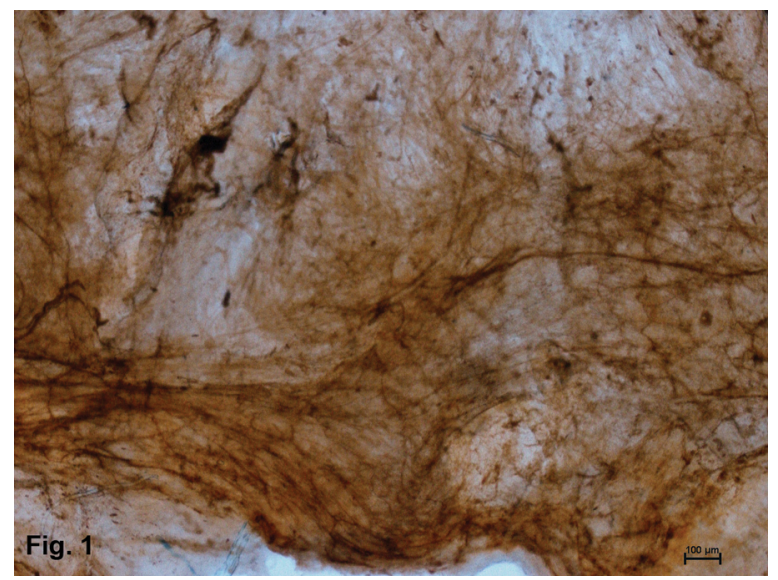

Figure 1. Cholinergic fibres in the mitral valve. The "parietal" part is at the bottom. Acetylcholine esterase (AChE) method.

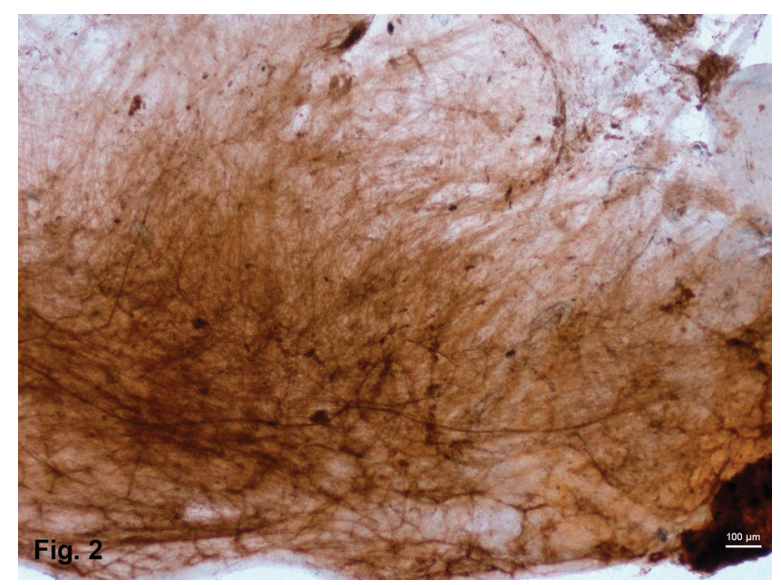

Figure 2. Tricuspid valve - the arrangement of cholinergic fibres. The "parietal" part is at the bottom, the free part of the cusps at the top. Acetylcholine esterase (AChE) method.

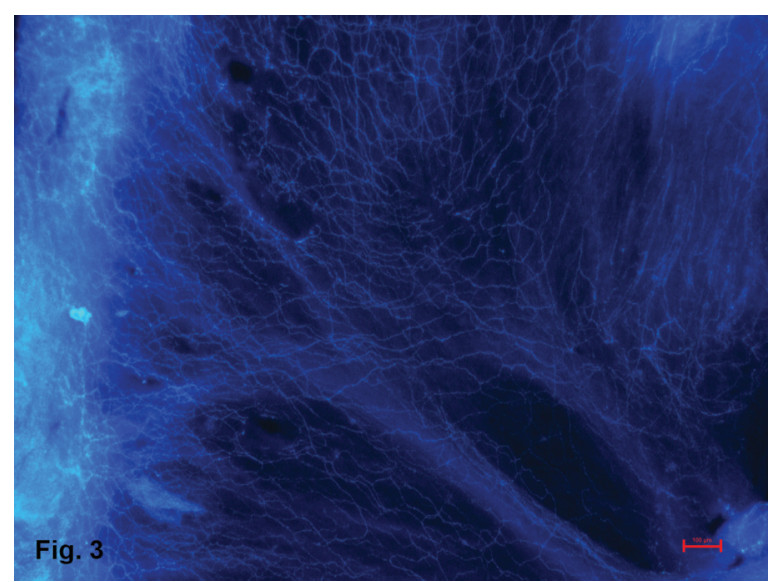

Figure 3. Adrenergic fibres in the mitral valve. The "parietal" part is on the left side. The initial parts of the tendinous cords on the right side. De la Torre method.

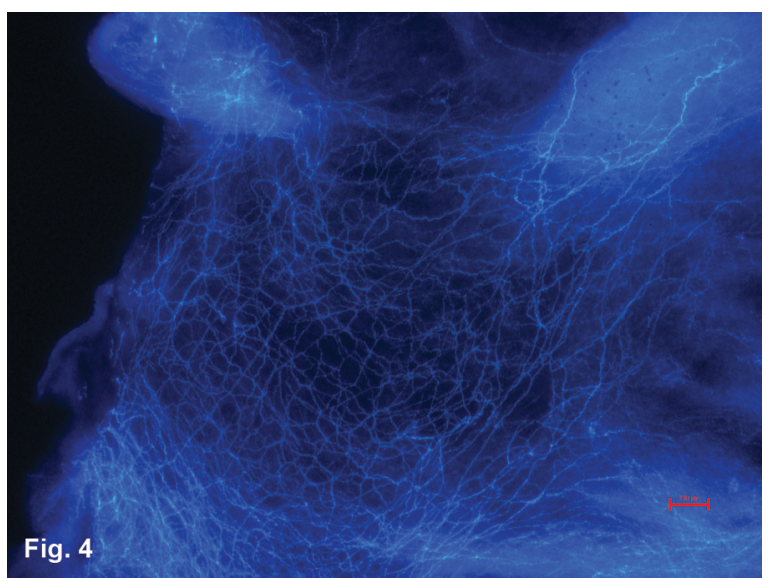

Figure 4. Adrenergic fibres in the tricuspid valve. The "parietal" part is on the left side. The initial parts of the tendinous cords on the right side. De la Torre method.

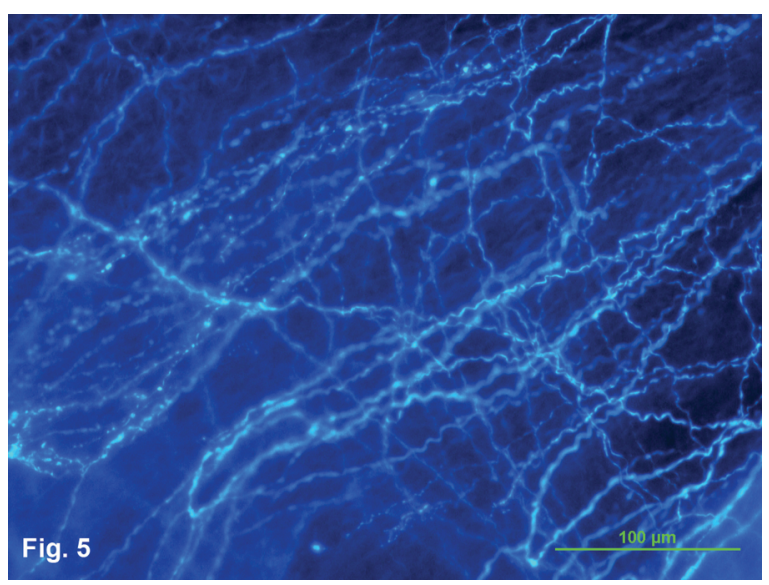

Figure 5. Adrenergic fibres in the initial parts of the tendinous cord. De la Torre method.

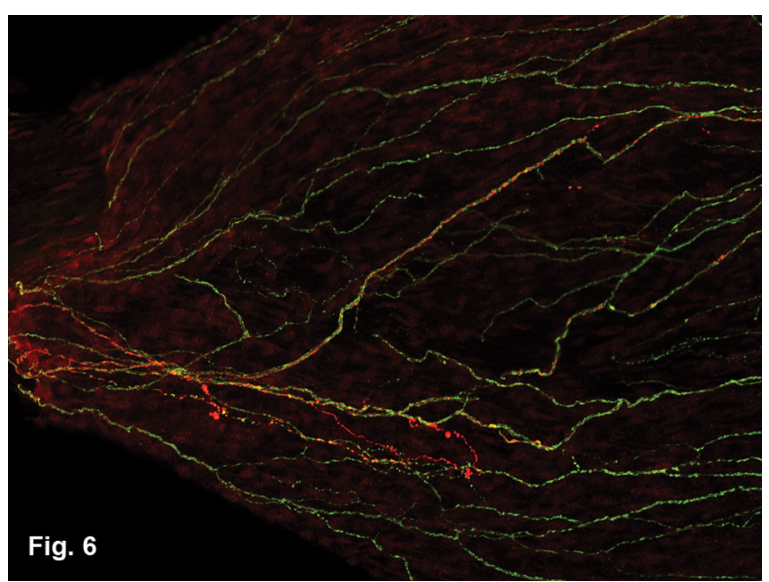

Figure 6. Colocalisation of fibres containing dopamine beta hydroxylase (DBH; green colour) and vesicular acetylcholine transporter (VAChT; red colour) in the distal part of the inferior cusp (anterior) of the mitral valve and the initial part of the tendinous cord. Double immunocytochemical staining. 
the nerve fibres in mice, guinea pigs and humans [16, 23] did not reach the free edges of the cusps. The opposite situation was observed in the innervation of the rat heart [25]. We can therefore assume that the density of the plexus in the distal portion depends on the kind of species, while it is an individual feature within a given species.

As observed in the chinchillas studied, some nerve fibres from the valve cusps connect with the plexus on the tendinous cords and papillary muscles. A similar situation exists in the human heart [16]. This is explained by other study results $[1,10,14]$ on the adrenergic innervation of the atrioventricular valves in the guinea pig. The authors demonstrated the presence of the two main plexuses: a) a larger, dense plexus located in the basal part of the valve cusps, along the annulus fibrosus, with branches to the central and marginal area of the cusps, b) a smaller one, moving upward from the tendinous cords and connecting with the distal region of the larger plexus.

The studies conducted by Ahmed et al. [1] proved that the larger plexus begins in the atria and the smaller in the ventricles, through the papillary muscles to tendinous cords. This is fairly obvious given the results of the studies conducted by Pauziene et al. [20]. The author has indicated the presence of nerve fibres and cells in rabbit hearts (also within the endocardium), immunoreactive for choline acetyl transferase (ChAT), neuronal nitric oxidase (nNOS) and both ChAT/nNOS and ChAT/tyrosine hydroxylase (TH).

The study showed that the adrenergic network was more strongly represented in the atrioventricular valves of chinchillas. Similar findings were presented by Borin et al. [2]. This is interesting because, based on the results of works on the innervation of the heart conduction system, the cholinergic network is more strongly represented $[3,18,19]$.

The immunocytochemical tests of recent years aimed to detect various kinds of neurotransmitters in cells and nerve fibres in the hearts of different species. Today, it is known that, in addition to the main neurotransmitters (AChE and NA), there are also NOS, NPY, TH, calcitonin gene related peptide (CGRP), and other neuropeptides $[3,9,11,15,17,21,22]$. The authors pay attention to the colocalisation relationships between various neurotransmitters. Also, our results showed such a relationship with respect to VAChT and DBH in the peripheral parts of the valve cusps and in tendinous cords. Taking into account the data on various subpopulations of cells within the heart, such colocalisation indicates the presence of cholinergic-adrenergic cells. Also in humans, the cusps of the atrioventricular valves demonstrate the presence of acetylcholine esterase or TH and NPY in nerve endings [16].

The cardiac plexuses are the source of autonomous fibres in the cardiac atrioventricular valves. They contain neurochemically and functionally diverse populations of neurons. Cholinergic neurons are the largest group. Another large group comprises neurons of a dual cholinergic-adrenergic nature. There are also sub-populations of cells with a different neurochemical nature [12]. Neurotransmitters of cardiac neurons include both classic neurotransmitters (acetylcholine and catecholamine pathway enzymes) and a number of neuropeptides, such as NOS, NPY, cocaine- and amphetamine-related transcript peptide, pituitary adenylate cyclase-activated peptide, nicotinamide adenine dinucleotide phosphatase $[11,12,15,18,21]$.

\section{CONCLUSIONS}

The study showed the presence of both cholinergic and adrenergic nerve fibres on all cusps of both valves. It was confirmed by double immunocytochemical staining for expression of VAChT and DBH. The adrenergic innervation of valves is more strongly represented that cholinergic one.

\section{REFERENCES}

1. Ahmed A, Johansson O, Folan-Curran J. Distribution of PGP 9.5, TH, NPY, SP and CGRP immunoreactive nerves in the rat and guinea pig atrioventricular valves and chordae tendineae. J Anat. 1997; 191 (Pt 4): 547-560, indexed in Pubmed: 9449074.

2. Borin C, Vanhercke D, Weyns A. Innervation of the atrioventricular and semi-lunar heart valves: a review. Acta Cardiol. 2006; 61(4): 463-469, doi: 10.2143/ /AC.61.4.2017309, indexed in Pubmed: 16970058.

3. Crick SJ, Anderson RH, Ho SY, et al. Localisation and quantitation of autonomic innervation in the porcine heart II: endocardium, myocardium and epicardium. J Anat. 1999; 195 (Pt 3): 359-373, indexed in Pubmed: 10580851.

4. De Biasi S, Vitellaro-Zuccarello L, Blum I. Histochemical and ultrastructural study on the innervation of human and porcine atrio-ventricular valves. Anat Embryol (Berl). 1984; 169(2): 159-165, indexed in Pubmed: 6742455.

5. De la Torre JC. An improved approach to histofluorescence using the SPG method for tissue monoamines. J Neurosci Methods. 1980; 3(1): 1-5, indexed in Pubmed: 6164878.

6. Fenoglio JJ, Wit AL, Bassett AL, et al. Canine mitral complex. Ultrastructure and electromechanical properties. Circ Res. 1972; 31(3): 417-430, indexed in Pubmed: 5057021.

7. Gienc J. The application of histochemical method in the anatomical studies on the parasympathetic ganglia and 
nerve bundles of postganglionic axons in the sublingual region of some mammals. Zool Pol. 1977; 26: 187-192.

8. Folan-Curran J, Wang YF, Jew JY, et al. The terminal innervation patterns in young and old guinea pig heart valves: a quantitative analysis using acetylcholinesterase staining. Exp Gerontol. 1994; 29(5): 543-552, indexed in Pubmed: 7530210.

9. Hoover DB, Ganote CE, Ferguson SM, et al. Localization of cholinergic innervation in guinea pig heart by immunohistochemistry for high-affinity choline transporters. Cardiovasc Res. 2004; 62(1): 112-121, doi: 10.1016/j. cardiores.2004.01.012, indexed in Pubmed: 15023558.

10. Jew JY, Fink CA, Williams TH. Tyrosine hydroxylase- and nitric oxide synthase-immunoreactive nerve fibers in mitral valve of young adult and aged Fischer 344 rats. J Auton Nerv Syst. 1996; 58(1-2): 35-43, indexed in Pubmed: 8740657

11. Kukanova B, Mravec B. Complex intracardiac nervous system. Bratisl Lek Listy. 2006; 107(3): 45-51, indexed in Pubmed: 16796123.

12. Kuder T, Nowak E. Autonomic cardiac nerves: literature review. Folia Morphol. 2015; 74(1): 1-8, doi: 10.5603/ /FM.2015.0003, indexed in Pubmed: 25792389.

13. Kumar SD, Tay SS. Changes in peptidergic nerves in the atrioventricular valves of streptozotocin-induced diabetic rats: a confocal microscopy study. Anat Rec. 2000; 258(3): 277-285, indexed in Pubmed: 10705348.

14. Lipp W, Rodin M. The adrenergic nerve plexuses of cardiac valves. Acta Anat (Basel). 1968; 69(3): 313-326, indexed in Pubmed: 5673451.

15. Lovasova K, Kluchova D, Bolekova A, et al. Distribution of NADPH-diaphorase and AChE activity in the anterior leaflet of rat mitral valve. Eur J Histochem. 2010; 54(1): 25-29, doi: 0.4081/ejh.2010.e5, indexed in Pubmed: 20353912.

16. Marron K, Yacoub MH, Polak JM, et al. Innervation of human atrioventricular and arterial valves. Circulation. 1996; 94(3): 368-375, indexed in Pubmed: 8759078.
17. Olsen LH, Mortensen $K$, Martinussen $T$, et al. Increased NADPH-diaphorase activity in canine myxomatous mitral valve leaflets. J Comp Pathol. 2003; 129(2-3): 120-130, indexed in Pubmed: 12921717.

18. Pauza DH, Rysevaite $\mathrm{K}$, Inokaitis $\mathrm{H}$, et al. Innervation of sinoatrial nodal cardiomyocytes in mouse. A combined approach using immunofluorescent and electron microscopy. J Mol Cell Cardiol. 2014; 75: 188-197, doi: 10.1016/j. yjmcc.2014.07.016, indexed in Pubmed: 25101952.

19. Pauza DH, Rysevaite-Kyguoliene K, Vismantaite J, et al. A combined acetylcholinesterase and immunohistochemical method for precise anatomical analysis of intrinsic cardiac neural structures. Ann Anat. 2014; 196(6): 430-440, doi: 10.1016/j.aanat.2014.08.004, indexed in Pubmed: 25262932.

20. Pauziene N, Alaburda P, Rysevaite-Kyguoliene K, et al. Innervation of the rabbit cardiac ventricles. J Anat. 2016; 228(1): 26-46, doi: 10.1111/joa.12400, indexed in Pubmed: 26510903.

21. Shoba T, Tay S. Nitrergic and peptidergic innervation in the developing rat heart. Anat Embryol. 2000; 201(6): 491-500, doi: 10.1007/s004290050336, indexed in Pubmed: 10909903.

22. Singh S, Johnson PI, Javed A, et al. Monoamine- and histamine-synthesizing enzymes and neurotransmitters within neurons of adult human cardiac ganglia. Circulation. 1999; 99(3): 411-419, doi: 10.1161/01.cir.99.3.411, indexed in Pubmed: 9918529.

23. Smith RB. Intrinsic innervation of the atrioventricular and semilunar valves in various mammals. J Anat. 1971; 108(Pt 1): 115-121, indexed in Pubmed: 5543206.

24. Sonnenblick EH, Napolitano LM, Daggett WM, et al. An intrinsic neuromuscular basis for mitral valve motion in the dog. Circ Res. 1967; 21(1): 9-15, doi: 10.1161/01. res.21.1.9, indexed in Pubmed: 6028860.

25. Williams TH, Folan JC, Jew JY, et al. Variations in atrioventricular valve innervation in four species of mammals. Am J Anat. 1990; 187(2): 193-200, doi: 10.1002/ /aja.1001870208, indexed in Pubmed: 2301279. 